\title{
-NOTES-
}

\section{A FORMULA FOR THE SOLUTION OF AN ARBITRARY ANALYTIC EQUATION*}

\section{By D. R. BLASKETT AND H. SCHWERDTFEGER (University of Adelaide)}

In this note a proof will be given of a formula which has been stated without proof by E. Schröder. ${ }^{1}$ The fact that he has expressed some doubts as to its general validity may have caused it to fall into oblivion although it seems to be of some theoretical and practical interest, like several other results of Schröder's to be mentioned below. Since moreover the true nature and simplicity of the formula is rather concealed in Schröder's discussion, it may be worth while to enter into the matter again.

The formula in question is a consequence of the following theorem which at once describes its exact realm of validity:

THEOREM I. Let $w=f(z)$ be an analytic function, regular in a domain $\Delta$ of the complex $z$-plane. Let $\alpha$ be an interior point of $\Delta$ and a simple root of $f(z)$;

$$
f(\alpha)=0, \quad f^{\prime}(\alpha) \neq 0 .
$$

Further let $z_{0}$ be a point in $\Delta$ "not too far from" $\alpha$ (in practice $z_{0}$ is a first rough approximation to the root $\alpha)$. Then, denoting by $z=f^{-1}(w)$ the inverse function of $f(z)$ one has

$$
\alpha=\sum_{\nu=0}^{\infty}(-1)^{\nu} \frac{f\left(z_{0}\right)^{\nu}}{\nu !}\left(\frac{d^{\nu} f^{-1}(w)}{d w^{\nu}}\right)_{w=f\left(z_{0}\right)}=\exp \left(-f\left(z_{0}\right) \frac{d f^{-1}(w)}{d w}\right)_{w=f\left(z_{0}\right)}
$$

where the exponential function operates symbolically on the differential symbol.

In this form the theorem is a corollary to the main theorem on the analyticity of the inverse of an analytic function. To prove (1) we make use of the fact that because $f^{\prime}(\alpha) \neq 0$ the function $f(z)$ is simple (schlicht) in a certain neighborhood of the point $z=\alpha .^{2}$ Hence the inverse $f^{-1}(w)$ exists in a circle $K$ of radius $\rho>0$ round the point $w=0$. Moreover it is analytic in $K$. Thus we may choose $z_{0}$ in a neighborhood of $\alpha$ such that a circle $k$ round $w_{0}=f\left(z_{0}\right)$ (in the $w$-plane) contains 0 and is wholly contained in $K$. In $k$ the function $f^{-1}(w)$ is given by its power series

$$
f^{-1}(w)=\sum_{\nu=0}^{\infty} \frac{1}{\nu !}\left(\frac{d^{\nu} f^{-1}(\omega)}{d \omega^{\nu}}\right)_{\omega=f\left(z_{0}\right)} \cdot\left(w-f\left(z_{0}\right)\right)^{\nu}
$$

whence for $w=0$ follows the formula (1). Evidently, for $z_{0}$, any point $z$ near $\alpha$ can be chosen for which $w=f(z)$ lies in a circle of radius $\rho / 2$ round 0 .

To obtain Schröder's formula we introduce the operators $\delta^{\mu}(\mu=0,1,2, \cdots)$ defined as follows:

* Received Oct. 30, 1944.

${ }^{1}$ E. Schröder, Über unendlich viele Algorithmen zur Auflösung der Gleichungen, Mathematische Annalen 2, 317-365 (1870); in particular cf. $\$ \$ 4$ and 5 of this paper.

2 Cf. G. Julia, Principes géométriques d'analyse, Première Partie, Paris 1930, p. 16-17. 


$$
\delta^{0} f(z)=\frac{1}{f^{\prime}(z)}, \quad \delta^{1} f(z)=\frac{1}{f^{\prime}(z)} \frac{d}{d z}\left(\frac{1}{f^{\prime}(z)}\right), \quad \delta^{\nu} f(z)=\frac{1}{f^{\prime}(z)} \frac{d}{d z}\left(\delta^{\nu-1} f(z)\right) .
$$

Then

$$
\delta^{1} f(z)=\left(\frac{d^{2} f^{-1}(w)}{d w^{2}}\right)_{w=f(z)}, \quad \delta^{\nu} f(z)=\left(\frac{d^{v+1} f^{-1}(w)}{d w^{v+1}}\right)_{w=f(z)}
$$

which is easily shown by induction. Therefore the expansion (1) is identical with

$$
\begin{aligned}
\alpha & =z_{0}+\sum_{\nu=1}^{\infty}(-1)^{\nu} \frac{f\left(z_{0}\right)^{\nu}}{\nu !}\left(\delta^{n-1} f(z)\right)_{z=z_{0}} \\
& =z_{0}-f\left(z_{0}\right) \frac{1}{f^{\prime}\left(z_{0}\right)}-\frac{f\left(z_{0}\right)^{2}}{2 !} \frac{f^{\prime \prime}\left(z_{0}\right)}{f^{\prime}\left(z_{0}\right)^{3}}+\frac{f\left(z_{0}\right)^{3}}{3 !} \frac{f^{\prime}\left(z_{0}\right) f^{\prime \prime \prime}\left(z_{0}\right)-3 f^{\prime \prime}\left(z_{0}\right)^{2}}{f^{\prime}\left(z_{0}\right)^{5}}+\cdots
\end{aligned}
$$

This was not the way in which the formula (2) was actually discovered. It appeared rather as a plausible consequence when dealing with another method of approximate solution of an analytic equation. Although no proof could be given on the basis of this method, it turns out to be of some interest here as it shows that the partial sums of the infinite series (1) or (2), i.e., the expressions

$$
\Phi_{n}(z)=\sum_{\nu=0}^{n}(-1)^{\nu} \frac{f(z)^{\nu}}{\nu !}\left(\frac{d^{\nu} f^{-1}(w)}{d w^{\nu}}\right)_{w=f(z)}=\sum_{\nu=0}^{n}(-1)^{\nu} \frac{f(z)^{\nu}}{\nu !} \delta^{\nu-1} f(z)
$$

can be used for an iterative approximation of the root $\alpha$;

THEOREM II. For each $n=1,2, \cdots$ the recurring sequence

$$
\alpha_{0}=z_{0}, \quad \alpha_{m}=\Phi_{n}\left(\alpha_{m-1}\right), \quad(m=1,2, \cdots)
$$

has the root $\alpha$ as its limit and then also

$$
\Phi_{n}(\alpha)=\alpha, \Phi_{n}^{\prime}(\alpha)=0, \cdots, \Phi_{n}^{(n)}(\alpha)=0 .
$$

This is one of the results of Schröder who has discussed in detail (1.c.) several such iterative algorithms ("Algorithmen erster Art") and has thus obtained formulae of considerable practical interest. ${ }^{3} \mathrm{We}$ propose here another treatment of the problem of iterative approximation which leads immediately to a proof of Theorem II.

We make use of the fact that if $\alpha$ is a simple root of the equation $f(z)=0$, a function $\phi(z)$ can be found for which $\alpha$ is an attractive fixed point, i.e.,

$$
\phi(\alpha)=\alpha, \quad\left|\phi^{\prime}(\alpha)\right|<1 .
$$

Then the recurring sequence $\alpha_{0}, \alpha_{1}=\phi\left(\alpha_{0}\right), \alpha_{2}=\phi\left(\alpha_{1}\right), \cdots$, if $\alpha_{0}$ is not too far from $\alpha$, is convergent and has $\alpha$ as its limit. To strengthen the convergence we may replace the inequality in (5) by the condition $\phi^{\prime}(\alpha)=0$. Such a function $\phi(z)$, involving an arbitrary function $h(z)$, is for instance

${ }^{3}$ In particular the reader's attention may be called to the formulae $\left(A_{1}^{\lambda}\right)$ and $\left(B_{2}^{\lambda}\right)$ in $\$ 12$ (p. 352) of Schröder's paper. These formulae are very expedient for the computation of $n$th roots if a high degree of accuracy (e.g. more than 20 correct figures) is required. A similar algorithm has recently been given by V. A. Bailey in a brief expository article Prodigious calculation, Australian Journal of Science, 3, 78-80 (1941), by which our attention has been drawn to the present subject.

4 Cf. G. Julia, loc. cit., p. 23. 


$$
\phi(z)=z+f(z) \frac{f(z) h(z)-1}{f^{\prime}(z)} .
$$

Thus we may impose more rigid conditions further strengthening the convergence, viz.,

$$
\phi^{\prime}(\alpha)=0, \phi^{\prime \prime}(\alpha)=0, \cdots, \phi^{(n)}(\alpha)=0,
$$

where $n$ is any positive integer. ${ }^{5}$

A function $\phi(z)$ satisf ying the conditions (5) and (6) can be obtained in the following way. The conditions (6) will be satisfied if the derivative of $\phi(z)$ appears in the form

$$
\phi^{\prime}(z)=(f(z))^{n} g(z) f^{\prime}(z),
$$

the undetermined function $g(z)$ being regular at $z=\alpha$. It remains to adapt $g(z)$ to the condition $\phi(\alpha)=\alpha$. One has

$$
\phi(z)=\int(f(z))^{n} g(z) f^{\prime}(z) d z=\int w^{n} g\left(f^{-1}(w)\right) d w
$$

whence, by repeated integration by parts, it follows that

$$
\phi(z)=n ! \sum_{\nu=0}^{n} \frac{(-1)^{\nu}}{(n-\nu) !}(f(z))^{n-\nu} g_{\nu+1}(z)
$$

where $g_{\mu}(z)$ is the $\mu$-fold iterated indefinite integral of $g\left(f^{-1}(w)\right)$ for $w=f(z)$. Thus, for $z=\alpha$ one has

$$
\phi(\alpha)=(-1)^{n} n ! g_{n+1}(\alpha) .
$$

Therefore

$$
g_{n+1}(z)=\frac{(-1)^{n}}{n !} z
$$

will give a function $\phi(z)$ which has all the desired properties. In this way one obtains the function $\Phi_{n}(z)$ of (3), and it is evident that this function has the properties stated in Theorem II.

5 From a letter of Professor V. A. Bailey we have learnt (in May 1941) that this problem has been dealt with in some special cases by E. Netto in his Vorlesungen über Algebra vol. I, Teubner, Leipzig, 1896, p. 300. In the same letter Bailey has given an elegant solution of the problem which, however, does not suit our present purpose. Further he has drawn our attention to the paper by L. Sancery, De la méthode des substitutions successives pour le calcul des racines des équations, Nouvelles Annales d. Math. (2) 1, 305-315 (1862), which, however, was not accessible to us.

\section{THE CAPACITY OF TWIN CABLE*}

By J. W. CRAGGS ANd C. J. TRANTER (Military College of Science, Stoke-on-Trent, England)

1. Introduction. The problem of determining the capacity of two long parallel cylindrical conductors can be easily solved by the use of a conformal transformation. ${ }^{1}$ A simple extension of the method gives the result for the case in which each conductor

* Received April 16, 1945.

${ }^{1}$ F. B. Pidduck, $A$ treatise on electricity, Cambridge University Press, 1916, p. 77. 